\title{
Analytical solutions for black-hole critical behaviour
}

\author{
1,2 Tomohiro Harada * and ${ }^{3}$ Ashutosh Mahajan $\dagger$ \\ ${ }^{1}$ Department of Physics, Rikkyo University, Toshima, Tokyo 171-8501, Japan, \\ ${ }^{2}$ Department of Physics, Kyoto University, Kyoto 606-8502, Japan, \\ ${ }^{3}$ Tata Institute for Fundamental Research, Homi Bhabha Road, Mumbai 400005, India
}

(Dated: January 10, 2019)

\begin{abstract}
Dynamical Einstein cluster is a spherical self-gravitating system of counterrotating particles, which may expand, oscillate and collapse. This system exhibits critical behaviour in its collapse at the threshold of black hole formation. It appears when the specific angular momentum of particles is tuned finely to the critical value. We find the unique exact self-similar solution at the threshold. This solution begins with a regular surface, involves timelike naked singularity formation and asymptotically approaches a static self-similar cluster.
\end{abstract}

PACS numbers: 04.70.Bw, 04.20.Dw, 04.20.Jb

General relativistic numerical simulation (numerical relativity) has revealed critical phenomena at the threshold of black hole formation in self-gravitating systems [1]. When a parameter $p$, which parametrises a generic oneparameter family of initial data sets, is tuned to the critical value $p^{*}$, there appears a self-similar solution, which is called a critical solution. Beyond this value, the collapse ends in a black hole, its mass $M_{\mathrm{BH}}$ obeying the power law $M_{\mathrm{BH}} \propto\left|p-p^{*}\right|^{\gamma}$, where $\gamma$ is called a critical exponent. The critical behaviour is well described in terms of the behaviour of solutions around a self-similar solution with a single unstable mode [2]. In this approach, self-similar solutions with regularity conditions are numerically found and a self-similar solution is numerically shown to be with a single, linearly unstable mode. See [3] for a recent review of critical phenomena. Apparently, there still is a huge gap between numerical simulation and linear stability analysis. Moreover, one could suspect unresolved fine structure at the threshold because almost all results have been based on numerics with finite accuracy (cf. [4]).

Here we show that there is a system where we can discuss critical phenomena in an analytical and exact manner. This is the spherical system of counterrotating particles, first introduced by Einstein [5] and later generalised to a dynamical case $[\underline{6}, 7,6]$. dinate $r$ comoving to the radial motion of each shell, the line element in this spacetime is given by

$$
d s^{2}=-e^{2 \nu(t, r)} d t^{2}+e^{2 \psi(t, r)} d r^{2}+R^{2}(t, r) d \Omega^{2},
$$

where $d \Omega^{2}$ is the line element on the two-dimensional unit sphere. The Einstein equations and conservation law reduce

$$
\nu^{\prime}=-\frac{1}{h(r, R)} \frac{\partial h(r, R)}{\partial R} R^{\prime}, \quad e^{2 \psi}=\frac{\left(R^{\prime}\right)^{2} h^{2}(r, R)}{1+2 E(r)},
$$

\footnotetext{
*Electronic address:harada@rikkyo.ac.jp

${ }^{\dagger}$ Electronic address:ashutosh@tifr.res.in
}

and

$$
\dot{R}^{2} e^{-2 \nu}=-1+\frac{2 M(r)}{R}+\frac{1+2 E(r)}{h^{2}(r, R)},
$$

where ${ }^{\circ} \equiv \partial / \partial t$ and ${ }^{\prime} \equiv \partial / \partial r, M(r)$ and $2 E(r)>-1$ are arbitrary functions corresponding to the Misner-Sharp mass and the specific energy, respectively, and $h(r, R)$ is given by

$$
h^{2}(r, R)=1+\frac{L^{2}(r)}{R^{2}},
$$

where $L(r)$ is the specific angular momentum of counterrotating particles. The energy density is

$$
\epsilon=\frac{M^{\prime}}{4 \pi R^{2} R^{\prime}} .
$$

The motion of each shell is governed by Eq. (3) or

$$
\frac{1}{2}\left(\frac{d R}{d \tau}\right)^{2}+U(r, R)=E(r),
$$

where $d \tau=e^{\nu} d t$ and

$$
U(r, R) \equiv-\frac{M(r)}{R}+\frac{(1+2 E(r)) L^{2}(r)}{2\left(R^{2}+L^{2}(r)\right)} .
$$

If we assume that the solution has a regular surface on which all regular metric functions and physical quantities are also analytic, this implies the Taylor-series expandability in terms of $R^{2}$. The arbitrary functions $M, E$ and $L^{2}$ then should be expanded as $M=M_{3} r^{3}+M_{5} r^{5}+\cdots$, $E=E_{2} r^{2}+E_{4} r^{4}+\cdots$, and $L^{2}=L_{4}^{2} r^{4}+L_{6}^{2} r^{6}+\cdots$, if we choose $r$ so that $r=R$ on the initial regular surface. $R$ is expanded as $R=R_{1}(t) r+R_{3} r^{3}+\cdots$ on a regular surface, and using Eq. (5), we have

$$
\epsilon(t, 0)=\frac{3 M_{3}}{4 \pi R_{1}(t)^{3}} .
$$

Observing the lowest order of Eq. (3), the evolution of $R_{1}(t)$ is given by

$$
\frac{1}{2}\left(\frac{d R_{1}}{d \tau}\right)^{2}=E_{2}+\frac{M_{3}}{R_{1}}-\frac{L_{4}^{2}}{2 R_{1}^{2}} .
$$


If $L_{4}^{2}>0$, we can find that $R_{1}(t)$ necessarily bounces and the neighbouring shell also does for sufficiently small $r>0$ [8, 9].

The case $L_{4}=0$ was studied in detail in [9]. For this case, from Eqs. (8) and (9), we find that an initially collapsing cloud inevitably form a central singularity after a finite proper time. As for the motion of the shell for sufficiently small $r>0$, it turns out that the quantity $\lambda \equiv \lim _{r \rightarrow 0} L(r) / M(r)=L_{6} / M_{3}$ becomes important from Eqs. (6) and (7). Figure 1 shows the shape of potentials for different values of $\lambda$. For $\lambda>4$, the region around $r=0$ necessarily bounces back, while, for $0 \leq \lambda<4$, the collapse continues to $R=0$. So, the end state of the collapse is a massless naked singularity or a black hole of finite mass depending on the value of $\lambda$. The critical value $\lambda^{*}$ is 4 . The mass scaling law and the critical exponent will be described elsewhere [10].

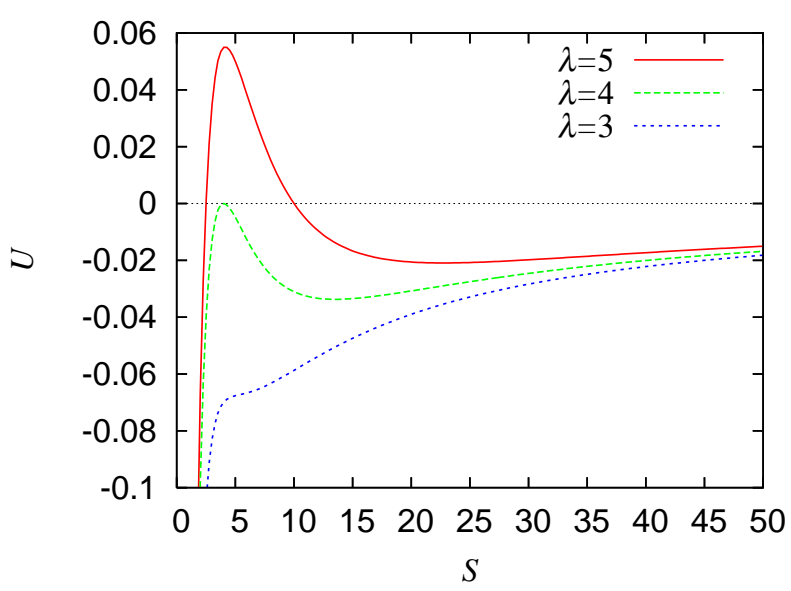

FIG. 1: The shape of the potential $U$ for $E=0$ and $L=\lambda M$ with $\lambda=3,4$ and 5 . The horizontal axis is $S \equiv R / M$.

The self-similarity (homothety) requires that there is a vector field $\mathbf{z}$ such that $\mathcal{L}_{\mathbf{z}} g_{a b}=2 g_{a b}$. This condition implies for the line element (10) that there is a coordinate system $(t, r)$ such that $\nu, \psi$ and $S \equiv R / r$ are functions of $z \equiv t / r$ [1]. For dynamical Einstein clusters, this means $E(r)=E, M(r)=\mu r$ and $L(r)=l r$, where $E, \mu$ and $l$ are constants. Then Eqs. (2) and (3) reduce the following closed autonomous system:

$$
\begin{aligned}
\frac{d \sigma}{d \ln |z|}-1 & =\frac{l^{2}}{S^{2}+l^{2}} \frac{1}{S}\left(\frac{d S}{d \ln |z|}-S\right), \\
e^{-2 \sigma}\left(\frac{d S}{d \ln |z|}\right)^{2} & =-1+\frac{2 \mu}{S}+\frac{(1+2 E) S^{2}}{S^{2}+l^{2}}
\end{aligned}
$$

where $\sigma \equiv \nu+\ln |z|$. We can eliminate $z$ from the above equations and get

$$
\frac{d e^{\sigma}}{d S}=\frac{l^{2}}{S\left(S^{2}+l^{2}\right)} e^{\sigma} \pm \frac{S^{2}}{S^{2}+l^{2}} \frac{1}{\sqrt{2(E-U(S))}}
$$

where the upper (lower) sign corresponds to the positive (negative) sign of $d S / d(\ln |z|)$ and

$$
U(S) \equiv-\frac{\mu}{S}+\frac{1}{2} \frac{(1+2 E) l^{2}}{S^{2}+l^{2}} .
$$

Equation (11) reduces

$$
\frac{1}{2}\left(\frac{d S}{d \xi}\right)^{2}+U(S)=E
$$

where $d \xi^{2} \equiv e^{2 \nu(z)} d z^{2}=e^{2 \sigma}(d \ln |z|)^{2}, \xi$ corresponding to the nondimensional proper time for the comoving observer. If we use $S$ as a coordinate in place of $t$, we get

$$
\begin{aligned}
d s^{2} & =e^{2 \zeta}\left[-\left(e^{\sigma} d \zeta \pm \frac{d S}{\sqrt{2(E-U(S))}}\right)^{2}+\frac{S^{2}+l^{2}}{(1+2 E) S^{2}}\right. \\
& \left.\times\left(S \mp e^{\sigma} \sqrt{2(E-U(S))}\right)^{2} d \zeta^{2}+S^{2} d \Omega^{2}\right],
\end{aligned}
$$

where $\zeta \equiv \ln r$ and $S$ plays a role of similarity variable. From Eq. (5),

$$
4 \pi \epsilon r^{2}=\frac{\mu}{S^{2}\left(S \mp e^{\sigma} \sqrt{2(E-U(S))}\right)} .
$$

If $R^{\prime}=0$ but $R \neq 0$, there appears shell-crossing singularity and the model gets invalid beyond there. This condition reduces the following equation:

$$
S \mp e^{\sigma} \sqrt{2(E-U(S))}=0 .
$$

The solution is shell-crossing free where $d S / d \ln |z|<0$.

When we rescale $t$ and $r$ as $\tilde{t}=a t$ and $\tilde{r}=a r$, we will get $\tilde{E}=E, \tilde{\mu}=\mu / a, \tilde{l}=l / a, \tilde{\sigma}=\sigma-\ln a$ and $\tilde{S}=S / a$ and the above equations are invariant. Hereafter, we fix this scaling so that $\mu=1$ and $l=\lambda$, in which $r$ coincides with the mass and is denoted as $m$. Note that we still have the freedom of rescaling as $\tilde{t}=a t$ because this only changes $z$ to $\tilde{z}=a z$ and Eqs. (10)-(11) are invariant. Except for this gauge freedom, there are a three-parameter family of self-similar solutions parametrised by $E, \lambda$ and the initial value for $\sigma=\sigma_{i}$ at $S=S_{i}$.

At the regular centre, $m / R$ vanishes or $S=\infty$. To assure the circular constant being $\pi, h^{2} /(1+2 E) \rightarrow 1$ as $S \rightarrow \infty$, implying $E=0$. Moreover, we assume that the cluster has a critical angular momentum, i.e., the righthand side of Eq. (11) has a double root, implying $\lambda=4$. Then, we have specified two parameters and have a oneparameter family of self-similar solutions. For $E=0$ and $\lambda=4$, the shape of the potential is shown in Fig. 1. Equation (14) then has essentially three solutions and all others are generated through time reversal or time translation of those solutions. The first one is a static solution at the top of the potential. For this case, we assume $\psi$ and $R$ have no dependence on $t$ but do not for 
$\nu$. This is because $\partial / \partial t$ may not coincide with a timelike Killing vector. Then, Eqs. (10)-(11) yield the following solution: $e^{2 \sigma}=c_{0}^{2}|z|, e^{2 \psi}=32$, and $S=4$, where $c_{0}$ is a constant and set to be unity, using the rescaling of $t$. The resulting metric is given by

$$
d s^{2}=-R d T^{2}+2 d R^{2}+R^{2} d \Omega^{2},
$$

where we have implemented the coordinate transformation: $d t^{2} /|t|=4 d T^{2}$ and $4 m=R$. The density $\epsilon$ is given by $4 \pi \epsilon=1 /\left(64 m^{2}\right)=1 /\left(4 R^{2}\right)$. This spacetime has a timelike naked singularity at the centre and suffers from a solid angle deficit so that the area of the sphere $R=$ const divided by the squared radius is not $4 \pi$ but $2 \pi$. It can be easily shown that static singular solutions, which were discovered in [12], all fall into this self-similar static solution after an appropriate coordinate transformation.

The remaining two solutions are dynamical. The second one is the solution, in which $S$ begins with infinity at $\xi=-\infty$, monotonically decreases and asymptotes 4 as $\xi$ increases to $\infty$. The third one is the solution, in which $S$ begins with 4 at $\xi=-\infty$, monotonically decreases to 0 at a finite value of $\xi$. For these two solutions, we can express $e^{\sigma}$ in terms of $S$ using only elementary functions as an integral of Eq. (12):

$e^{2 \sigma}=\frac{S^{2}}{S^{2}+16}\left(\frac{\sqrt{2 S}}{3}(S+12)+4 \sqrt{2} \ln \left|\frac{\sqrt{S}-2}{\sqrt{S}+2}\right|+A\right)^{2}$,

where $A$ is an arbitrary constant. This is actually a subclass of solutions whose expressions were given in terms of elementary functions in [9]. Only the second solution has a regular centre because $m / R$ is always equal to and larger than a quarter for the static solution and the third solution, respectively.

Let us concentrate on the second solution. Around the regular centre $S=\infty$, from Eq. (18), we have

$$
e^{\sigma}=\frac{\sqrt{2}}{3} S^{3 / 2}+4 \sqrt{2} S^{1 / 2}+A+O\left(S^{-1 / 2}\right) .
$$

Then, from Eqs. (10) and (11), we have for the lowest order term $S \approx(9 / 2)^{1 / 3} X^{2 / 3}|z|^{2 / 3}$ and $e^{\sigma} \approx X|z|$, as $|z| \rightarrow \infty$, where the constant $X$ can be set to be unity by rescaling $t$. This is exactly the behaviour of the regular centre: $R \propto m^{1 / 3}$ and $e^{\nu} \rightarrow 1$ at $m \rightarrow 0$. In this regime, $\xi=z+$ const, and $t$ coincides with the proper time at the regular centre. Equation (16) implies $4 \pi \epsilon_{0}(t) t^{2}=2 / 3$, where $\epsilon_{0}(t)$ is the density at the regular centre. The central density diverges to infinity at $t=0$, resulting in a central singularity. In other words, $t=0$ is characterised with the appearance of the central singularity. Let us assume the expandability for the density on the regular surface $t=$ const $<0$ :

$$
\epsilon=\sum_{i=0}^{\infty} \epsilon_{i}(t) R^{i}
$$

On this surface, we can write down $S=R / m$ as

$$
S=\left(\frac{4}{3} \pi \epsilon_{0} R^{2}\right)^{-1}\left(1+\frac{3}{\epsilon_{0}} \sum_{i=1}^{\infty} \frac{\epsilon_{i}}{i+3} R^{i}\right)^{-1} .
$$

Substituting Eqs. (20) and (21) into Eq. (16) and comparing both sides, we get $\epsilon_{1}=0, \epsilon_{2}=(160 / 3) \pi \epsilon_{0}^{2}$ and $\epsilon_{3}=16 \sqrt{2 / 3} \pi^{3 / 2} A \epsilon_{0}^{5 / 2}$. Therefore, the density around the regular centre can be expanded as

$$
\epsilon=\frac{1}{6 \pi t^{2}}+\frac{40}{27 \pi} \frac{R^{2}}{t^{4}}+\frac{4}{27 \pi} A \frac{R^{3}}{|t|^{5}}+O\left(R^{4}\right) .
$$

The density takes a local minimum at the centre. If we assume analyticity on the regular surface, $A=0$ is concluded and there no longer appear odd power terms of $R$ in the expansion. This analyticity requirement has been imposed for the identification of critical solutions [2]. So we will identify the second solution for $A=0$ with the unique threshold solution. Note that the analysis below is nevertheless also applicable to the case $A \neq 0$.

Let us see other physical properties of this solution. We can find from Eq. (18) that $e^{\sigma}$ can be zero. Let $\alpha(>4)$ such that $e^{\sigma}=0$ at $S=\alpha . \alpha(=4.8133 \cdots)$ is a root of the following transcendental equation:

$$
\sqrt{S}(S+12)=12 \ln \frac{\sqrt{S}+2}{\sqrt{S}-2} .
$$

Around there, from Eqs. (11) and (12), we can find $\mid S-$ $\left.\alpha|\propto| z\right|^{\beta}$ and $e^{\sigma} \propto|z|^{\beta}$ as $z \rightarrow 0$, where $\beta=\alpha^{2} /\left(\alpha^{2}+16\right)$ and hence $1 / 2<\beta<1$. In this regime, $\xi=\mp B|z|^{\beta}+$ const, where $B$ is a constant. This means that it takes only a finite proper time to reach $S=\alpha$ or $t=0$. Thus, $t=0$ is only a coordinate singularity and the solution can be extended regularly and uniquely beyond $t=0$. This is clear in Eq. (15), where there is no singularity at $S=\alpha$. The density profile at $t=0$ follows an exact power law $4 \pi \epsilon=1 /\left(\alpha R^{2}\right)$. Because of self-similarity, $z=0$ also corresponds to infinity $(|t|<\infty$ and $m=\infty)$. This really corresponds to the surface of infinite area $R=\infty$. It follows that the density falls off as $4 \pi \epsilon \approx 1 /\left(\alpha R^{2}\right)$ as $R \rightarrow \infty$. This behaviour is time-independent.

Each shell dynamically approaches the specific radius $S=4$ as $\xi \rightarrow \infty$. For $S \rightarrow 4$, from Eq. (18), we have $e^{\sigma} \approx-4 \ln (S-4)$. Then, from Eqs. (10) and (11), we have $(S-4) \approx e^{-C|z|^{1 / 2}}$ and $e^{\sigma}=4 C|z|^{1 / 2}$ as $|z| \rightarrow$ $\infty$, where $C>0$ is a constant. This means that the asymptotic solution is the static solution. In this regime, $\xi \approx 8 B|z|^{1 / 2}+$ const, and $\tau \approx-8 m \ln (S-4)$. Hence the collapse approaches the static solution with an infinite proper time.

Since $d S / d \ln |z|>0$ for $t<0$ for the threshold solution, we need to check whether there is a root in $(\alpha, \infty)$ of Eq. (17) or the following equation:

$$
\frac{2(S-4)}{\sqrt{S}\left(S^{2}+16\right)}\left|\frac{\sqrt{S}}{3}(S+12)+4 \ln \frac{\sqrt{S}-2}{\sqrt{S}+2}\right|=1 .
$$


When $S$ increases from $\alpha$ to $\infty$, the left-hand side increases from 0 , takes a maximum and decreases to $2 / 3$. This maximum value is $0.76412 \ldots$, well below unity. Since $d S / d \ln |z|<0$ for $t>0$, the threshold solution is free of shell-crossing.

Figure 2 shows the evolution of the density in terms of $S$ from Eq. (16). As time proceeds from $t=-\infty$ to $t=\infty, S$ monotonically decreases from $\infty$ to 4 and the density $\epsilon$ observed by a comoving observer monotonically increases from 0 to the value for the static solution $1 /\left(256 \pi m^{2}\right)$. It smoothly crosses $z=0$ at $S=\alpha$ and $\epsilon=1 /\left(4 \pi \alpha^{3} m^{2}\right)$. Figure 3 shows the ratio of the

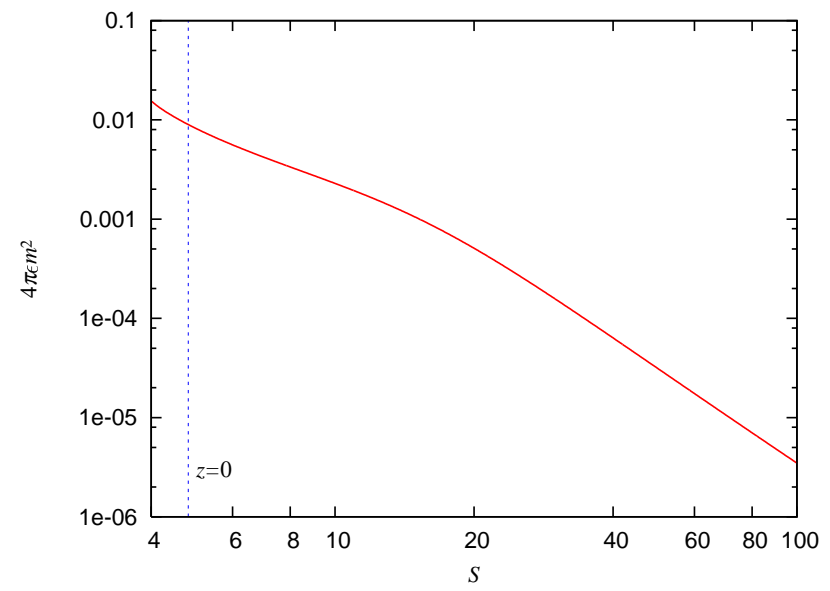

FIG. 2: The nondimensional density $4 \pi \epsilon m^{2}$ in terms of $S$ for the exact threshold solution.

local density $\epsilon$ to the averaged density inside the shell $m /\left(4 \pi R^{3} / 3\right)$, in which the horizontal axis is $1 / \sqrt{S}$. This ratio must be unity at the regular centre and $1 / \sqrt{S}$ is proportional to the area radius around the regular centre. We can see that the density takes a local minimum at the regular centre $S=\infty$ or $z=-\infty$, increases around the centre, takes a maximum and decreases outside. At spatial infinity $S=\alpha$ or $z=0$, this ratio becomes $1 / 3$, implying that $\epsilon$ falls off in proportion to $R^{-2}$. The ratio goes below $1 / 3$ as $z$ increases further and comes back to $1 / 3$ at $S=4$ or $z=\infty$, implying that $\epsilon$ again gets proportional to $R^{-2}$ as $t \rightarrow \infty$.

There is no trapped surface because $S=R / m>4$ is satisfied everywhere. So, the central singularity is naked. In fact, this spacetime is a member of solutions for which the causal structure is shown in Figure 1 of [12]. The central singularity is naked and timelike.

The general solutions of the dynamical Einstein cluster is exactly solved in terms of elliptic integrals using the mass-area coordinates [9, 13] and here the critical self-similar solution is uniquely obtained in terms of el- ementary functions. This system provides a tractable laboratory for studying how generic critical collapse approaches the threshold solution in both linear and nonlinear regimes.

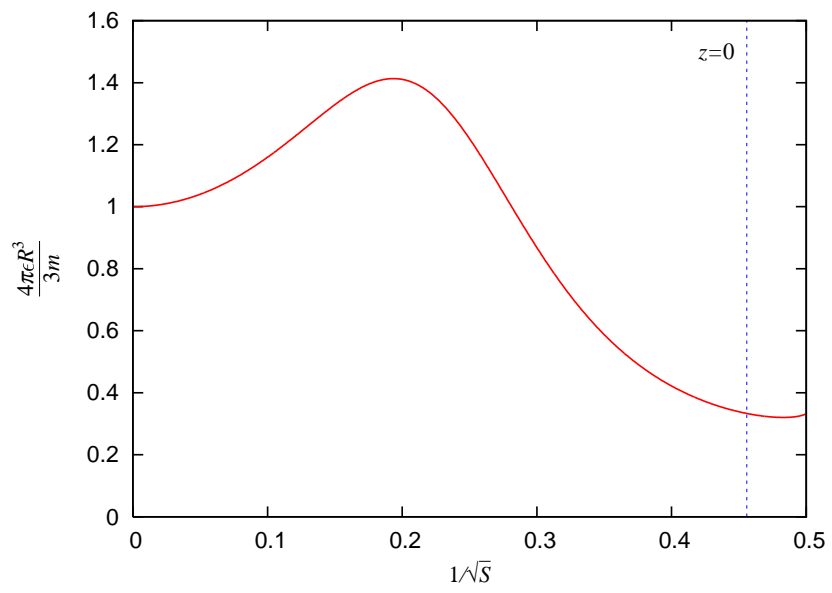

FIG. 3: $\left(4 \pi \epsilon R^{3}\right) /(3 m)$, the ratio of the local density to the averaged density interior to the shell in terms of $1 / \sqrt{S}$ for the exact threshold solution.

TH is very grateful to K. Nakao and H. Iguchi for helpful comments. This work was partly supported by the Grant-in-Aid for the 21st Century COE "Center for Diversity and Universality in Physics" and the Grant-inAid for Scientific Research Fund (Young Scientists (B) 18740144) of the Ministry of Education, Culture, Sports, Science and Technology of Japan.

[1] M. W. Choptuik, Phys. Rev. Lett. 70, 9 (1993).

[2] T. Koike, T. Hara, S. Adachi, Phys. Rev. Lett. 74, 5170 (1995).

[3] C. Gundlach, Physics Reports 376, 339 (2003).

[4] P. Bizoń, S. J. Szybka and A. Wasserman, Phys. Rev. D 69, 064014 (2004).

[5] A. Einstein, Ann. Math. 40, 922 (1939).

[6] B. K. Datta, Gen. Relat. Grav. 1, 19 (1970).

[7] H. Bondi, Gen. Relat. Grav. 2, 321 (1971).

[8] A. B. Evans, Gen. Relat. Grav. 8, 155 (1976).

[9] T. Harada, H. Iguchi and K. Nakao, Phys. Rev. D58, 041502(R) (1998).

[10] A. Mahajan, T. Harada, P.S. Joshi and K. Nakao, submitted to Prog. Theor. Phys.

[11] M. E. Cahill and A. H. Taub, Commun. Math. Phys. 21, 1 (1971).

[12] H. Kudoh, T. Harada and H. Iguchi, Phys. Rev. D62, 104016 (2000).

[13] G. Magli, Class. Quant. Grav. 15, 3215 (1998). 